\title{
f 377678 \\ 1999059771 \\ Contactless Growth of ZnSe Single Crystals by Physical Vapor Transport
}

\author{
Ching-Hua Su ${ }^{a,{ }^{*}}$, M. A. George ${ }^{b}$, W. Palosz ${ }^{a, 1}$, S. Feth ${ }^{a, 2}$ and S. L. Lehoczky

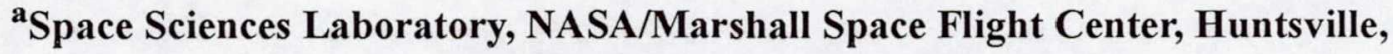 \\ Alabama, 35812 USA \\ ${ }^{b}$ Center for Materials and Surface Science, Department of Chemistry, University of \\ Alabama in Huntsville, Alabama, 35899 USA
}

\begin{abstract}
ZnSe crystals were grown by self-seeded physical vapor transport (PVT) technique in the horizontal configuration. The source materials were heat treated by $\mathrm{H}_{2}$ reduction to remove the oxide followed by baking under dynamic vacuum to adjust the source composition toward that of congruent sublimation. Contactless growth of $\mathrm{ZnSe}$ single crystals have been performed consistently using three different source materials. The crystals grew away from the wall during the later stage of the growth with large (110) facets tend to align parallel to the gravity direction. The SEM micrographs and the AFM images showed that large (110) terraces and steps dominate the as-grown facets. The measured residual gas pressures in the processed ampoules agree well among various source materials and the major components were $\mathrm{CO}$ and $\mathrm{H}_{2}$. No preferred growth direction was found. The one-dimensional diffusion model on the mass flux of a multi-species PVT system was employed to analyze the conditions for contactless growth. The calculated thermal profile for supersaturation is very close to the thermal profile measured inside the empty furnace bore in the region of contactless growth. The effects of convective flows in the vapor phase inside the ampoule on the growth processes are discussed.
\end{abstract}

\footnotetext{
* Corresponding author

${ }^{1}$ Universities Space Research Association

${ }^{2}$ Raytheon STX Corporation
} 